\title{
Effects of Caffeine on Crayfish Muscle Fibers
}

\author{
II. Refractoriness and factors \\ influencing recovery (repriming) \\ of contractile responses
}

\author{
DANTE J. GHIARANDINI, JOHN P. REUBEN, \\ LUGIEN GIRARDIER, GEORGE M. KATZ, \\ and HARRY GRUNDFEST
}

\begin{abstract}
From the Laboratory of Neurophysiology, Department of Neurology, College of Physicians and Surgeons, Columbia University, New York 10032. Dr. Chiarandini's present address is Instituto de Anatomia General, Facultad de Medicina, Buenos Aires, Argentina.

Dr. Girardier's present address is Institut de Physiologie, Ecole de Medecine, Geneva, Switzerland.
\end{abstract}

\begin{abstract}
ABSTRAGT When caffeine evokes a contraction, and only then, crayfish muscle fibers become refractory to a second challenge with caffeine for up to $20 \mathrm{~min}$ in the standard saline $\left(5 \mathrm{~mm} \mathrm{~K} \mathrm{~K}_{0}\right.$ ). However, the fibers still respond with contraction to an increase in $\mathrm{K}_{0}$, though with diminished tension. Addition of Mn slows recovery, but the latter is greatly accelerated during exposure of the fiber to high $\mathrm{K}_{0}$, or after a brief challenge with high $\mathrm{K}_{0}$. Neither the depolarization induced by the $\mathrm{K}$, nor the repolarization after its removal accounts for the acceleration, which occurs only if the challenge with $\mathrm{K}$ had itself activated the contractile system; acceleration is blocked when contractile responses to $\mathrm{K}$ are blocked by reducing the $\mathrm{Ca}$ in the bath or by adding $\mathrm{Mn}$. Recovery is accelerated by redistribution of intracellular $\mathrm{Cl}$ and by trains of intracellularly applied depolarizing pulses, but not by hyperpolarization. The findings indicate that two sources of Ca can be mobilized to activate the contractile system. Caffeine mobilizes principally the Ca store of the SR. Depolarizations that are induced by high $\mathrm{K}_{o}$, by transient efflux of $\mathrm{Cl}$, or by intracellularly applied currents mobilize another source of $\mathrm{Ca}$ which is strongly dependent upon the entry of Ca from the bathing medium. The sequestering mechanism of the SR apparently can utilize this second source of $\mathrm{Ga}$ to replenish its own store so as to accelerate recovery of responsiveness to a new challenge with caffeine.
\end{abstract}

\section{INTR ODUCTION}

This paper continues the report of a study of the chemical activation of contractions by caffeine that was begun in the accompanying paper. Some of 
the data of that work led to the conclusion that in the living crayfish muscle fiber caffeine acts intracellularly much as it does on the sarcotubular preparation of frog and rabbit muscle (Weber and Herz, 1968), primarily to inhibit the rapid $\mathrm{Ca}$ uptake by the Ca-sequestering system of the SR. This working hypothesis has been explored in considerable detail in the present study. This study takes advantage of the observation that crayfish muscle fibers which have responded to a challenge with caffeine become refractory to a second challenge, recovering their responsiveness fully only after 15-20 min. It was also observed that the refractoriness can be reduced or abolished by certain procedures. The refractoriness appears to be due to the depletion of $\mathrm{Ca}$ from its store in the SR and the duration presumably indicates the slow time course of replenishment of that store in the intact muscle fiber. The acceleration of the return of responsiveness is to a degree analogous with the "repriming" of $\mathrm{K}$ - and current-induced tensions in frog muscle (Hodgkin and Horowicz, 1960; Heistracher and Hunt, 1969), but in the present case the conditions that cause the repriming are very different.

The data on these conditions permit some inferences regarding the mechanisms of the repriming process. They indicate that the acceleration of repriming is effected by making available an additional source of $\mathrm{Ca}$, mainly or exclusively from the external medium, although the participation of an intracellular source of $\mathrm{Ca}$ is not ruled out.

\section{METHODS}

The methods employed in the present work were as described in the accompanying paper. Two types of control salines were employed, with the cations identical (200 mм Na, $5 \mathrm{~mm} \mathrm{~K}, 13.5 \mathrm{~mm} \mathrm{Ca}, 2 \mathrm{~mm}$ Tris). In one control saline the anion was $\mathrm{Cl}$, in the other the $\mathrm{Cl}$ was replaced with an impermeant anion, propionate. Unless otherwise stated both conditioning and testing challenges were made with $10 \mathrm{~mm}$ caffeine. The wash-in and washout times were each of the order of $4 \mathrm{sec}$. Controls are included in the illustrations of most of the experiments described.

R E S U L T S

\section{A. Refractoriness and Recovery of Responsiveness to Caffeine}

TIME COURSE OF REFRAGTORINESS The transient caffeine tension (see accompanying paper) can be elicited reproducibly an unlimited number of times in fibers bathed in normal saline, provided a sufficient interval of time elapses between exposures to caffeine. This interval is seldom less than 15 or greater than $20 \mathrm{~min}$. When caffeine is reapplied at shorter intervals than these the tensions are less than maximal. In the records of Fig. 1 the peak tension evoked by reapplying caffeine $2.5 \mathrm{~min}$ after an initial challenge was less than $10 \%$ of the control response. As the interval between two consecutive challenges was increased the second response increased, reaching its full amplitude when the interval was $20 \mathrm{~min}$ (bottom row). The graph of Fig. 1 
shows the time course of the recovery of another muscle fiber when two levels of caffeine concentrations (10 mm, solid circles; $40 \mathrm{~mm}$, open circles) were used. Since the higher concentration of caffeine evoked larger tensions (see accompanying paper, Fig. 2) the ordinate of the graph represents the tension expressed as percentage of the maximal response observed with each concentration. The fiber was challenged twice with $10 \mathrm{~mm}$ caffeine with an interval of $30 \mathrm{~min}$ between applications to ascertain that the control tension was reproducible (solid circle 1). A second application made 2.5 min later

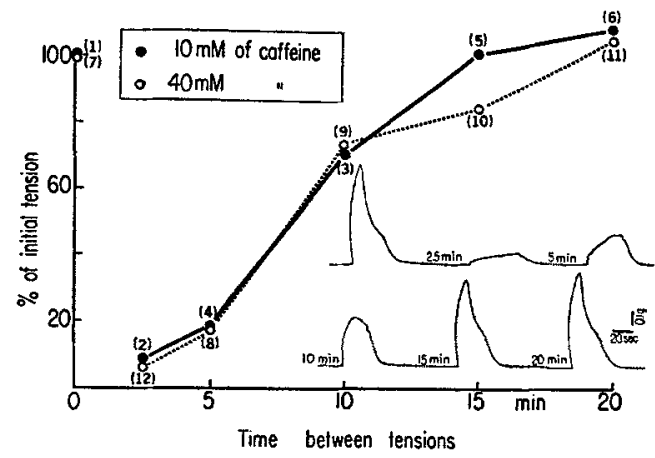

Figure 1. Recovery of responsiveness to caffeine. Insets, (upper row) A challenge with $10 \mathrm{~mm}$ caffeine produced an initial tension; the drug was removed and $2.5 \mathrm{~min}$ later the same concentration of caffeine produced only a small response. 5 min after washing out the drug, a third challenge induced a tension of about $30 \%$ of the original. The same procedure was repeated (lower row) for longer intervals between tensions $(10,15$, and $20 \mathrm{~min}$ ). Full recovery was obtained when the interval between two consecutive challenges was $20 \mathrm{~min}$. Graph, the time course of the recovery of responsiveness obtained with another fiber. The numbers indicate the sequence of successive applications of caffeine. Ordinate, tension expressed as percentage of the initial tension. After challenge 6 , the fiber was rested for $20 \mathrm{~min}$ in normal saline and then challenge 7, with $40 \mathrm{~mm}$ caffeine, induced a tension $40 \%$ larger than the control (No. 1) with $10 \mathrm{~mm}$ caffeine. The responses to subsequent challenges (8-12) with $40 \mathrm{~mm}$ caffeine are expressed as per cent of this new control.

(No. 2) resulted in a response of less than $10 \%$ of the control. 10 min later a third application (No. 3) evoked a tension about $70 \%$ of the control. Further applications were made with intervals of $5 \mathrm{~min}$ (No. 4), $15 \mathrm{~min}$ (No. 5), and $20 \mathrm{~min}$ (No. 6). This last challenge elicited a tension slightly larger than the control indicating full recovery of responsiveness. After resting the fiber for $20 \mathrm{~min}, 40 \mathrm{~mm}$ caffeine was applied and induced a tension (No. 7) 40\% larger than the one obtained with $10 \mathrm{~mm}$ caffeine. This was taken as control for the second part of the experiment. The procedure described above was repeated and the time course of the recovery of responsiveness was thus obtained. The course of the recovery of responsiveness was independent of these caffeine concentrations.

It was noted in the accompanying paper that the transience of tension 
during continued exposure to caffeine probably indicated the existence of a caffeine-insensitive Ca-sequestering system. The refractoriness demonstrated in Fig. 1 might therefore reflect the slowed reaccumulation of $\mathrm{Ca}$ in the primary caffeine-sensitive sequestering system of the SR as a consequence of competition with the second sequestering system.

DEVELOPMENT OF REFRACTORINESS In the experiment of Fig. 2 the duration of the initial exposure to caffeine $(10 \mathrm{~mm})$ was varied between 4 and $40 \mathrm{sec}$. In all cases the test application of caffeine was made $1.5 \mathrm{~min}$ after the

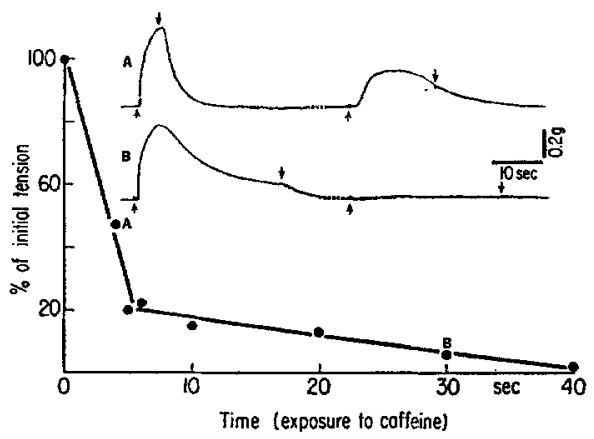

FIGURE 2. Effect of different exposure times to caffeine on the subsequent refractoriness. In this and subsequent figures each application and removal of caffeine is indicated by a pair of arrows. Inset $A$, tension elicited by exposing the fiber to $10 \mathrm{~mm}$ caffeine for 4 sec. The drug was removed and $90 \mathrm{sec}$ later, when caffeine was applied a second time, it induced a tension about 50\% of the first response. Small "wash-in" and "washout" artifacts are seen in the record. $B$, the fiber was exposed for $30 \mathrm{sec}$ to $10 \mathrm{~mm}$ caffeine. A second challenge was given $90 \mathrm{sec}$ after removing the drug and elicited a response less than $10 \%$ of the control. Note that in both cases the peak amplitudes of the first responses were similar, but only in $B$ was the course of the tension completed. Graph, a series of measurements on the same fiber with the exposure times to caffeine ranging from 4 to $40 \mathrm{sec}$. Points $A$ and $B$ are the data for the corresponding records. Most of the refractoriness to a second challenge develops in the first $6 \mathrm{sec}$ of the initial application of caffeine.

initial dose of caffeine had been washed out (arrows). Trace $A$ shows the tension elicited by exposing the fiber to caffeine for $4 \mathrm{sec}$. The tension evoked by the second challenge was almost $50 \%$ of the initial response. In $B$ the first exposure to caffeine lasted $30 \mathrm{sec}$ and during this time the contraction had almost completed its normal course. The testing challenge now induced almost no measurable tension. The graph of Fig. 2 was constructed from a series of such measurements on this fiber. It appears from these data that the process inducing the major phase of the refractoriness occurs during the first $6 \mathrm{sec}$ of an exposure of the fiber to $10 \mathrm{~mm}$ caffeine. It is interesting to note in this connection that the decay of spike-induced tension in the presence of caffeine (see accompanying paper, Fig. $12 \mathrm{G}$ ), which has been ascribed to the uptake of the elevated Ca by the second sequestering system, has a time constant of about 2-3 sec. 
INFLUENCE OF INCREASED Ca It might be expected that raising the external $\mathrm{Ca}$ would accelerate the recovery from refractoriness, by increasing the supply of intracellular Ca to relieve the "exhaustion" of the SR store following a challenge with caffeine. However, recovery is not significantly altered by increasing external $\mathrm{Ca}$ from $13.5-50 \mathrm{~mm}$ (Fig. 3). This experiment was done on a single fiber starting with $13.5 \mathrm{~mm} \mathrm{Ca}$ and following the procedure described for Fig. 1. After the seventh measurement in the standard solution the fiber was exposed to the saline containing $50 \mathrm{~mm} \mathrm{Ca}$ and the measurements were repeated. In the high $\mathrm{Ca}$ saline the tension evoked by caffeine

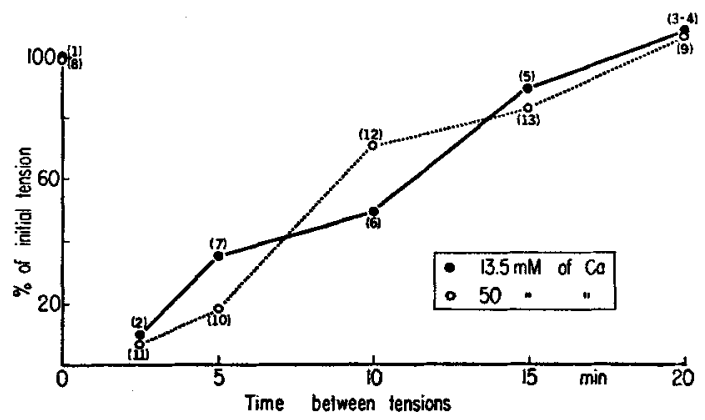

Figure 3. Recovery of responsiveness in the presence of 13.5 and $50 \mathrm{mM} \mathrm{Ca}$ in the bath. Experiments performed as described in Fig. 1. Numbers associated with the points show the sequence of the challenges. The control response (No. 8) was larger and more prolonged when $\mathrm{Ca}_{o}$ was raised to $50 \mathrm{~mm}$.

lasts longer and is about $25 \%$ higher than the corresponding tension obtained in the normal saline.

INFLUENCE of Mn It was not possible to carry out a similar set of measurements in a Ca-free medium, because in the absence of $\mathrm{Ca}$ the tensions evoked by successive challenges with caffeine decline rapidly (see accompanying paper, Fig. 7), even though the interval between the challenges is very long. Another procedure was employed to reduce $\mathrm{Ca}$ flux, taking advantage of the finding that $\mathrm{Mn}$ reduces $\mathrm{Ca}$ influx in barnacle muscle (Hagiwara and Nakajima, 1966) and in heart atrial muscle (Rougier et al., 1969). The upper row of Fig. 4 shows a series of caffeine-induced tensions in which the intervals between two consecutive challenges were increased progressively. The tension evoked $20 \mathrm{~min}$ after the previous challenge was slightly larger than the control, as is also seen in Figs. 1 and 3. Mn (20 mM) was introduced at the arrow (upper row). The next challenge with caffeine followed after 5 min (lower row) and subsequent challenges were made after progressively longer intervals. Recovery was much slower in the presence of $\mathrm{Mn}$. Even when the interval between two applications of caffeine was as long as $40 \mathrm{~min}$ the tension was somewhat lower than that for the control challenges. When the $\mathrm{Mn}$ was removed from the bath (arrow, lower row) recovery resumed its normal 
course. $20 \mathrm{~min}$ after washing out the $\mathrm{Mn}$ the test response to caffeine was higher than the last test response in the upper row.

EFFECT OF THE PRESENCE OR ABSENCE OF TENSION In order to cause refractoriness the caffeine must first induce tension. The upper row of Fig. 5 shows records of the tension evoked by the initial application of caffeine and of the very small tension induced when a second challenge was given $2.5 \mathrm{~min}$ later. The second row shows a sequence made 20 min later. Procaine $\left(10^{-3}\right.$

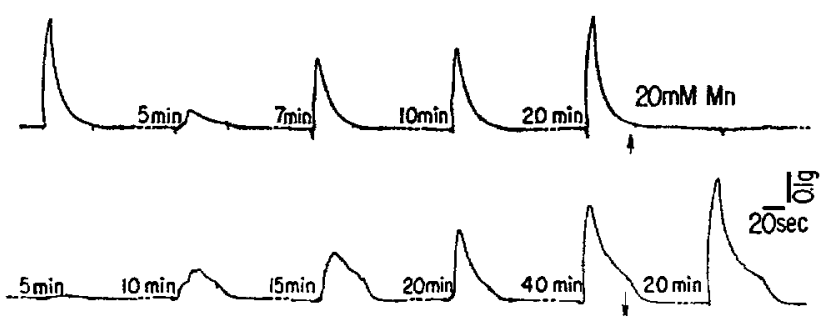

FIGURE 4. Effect of Mn on the recovery of responsiveness to caffeine. Upper row, control series. The first application of caffeine evoked a tension of about $0.4 \mathrm{~g}$. A second application $5 \mathrm{~min}$ later resulted in a tension of less than $20 \%$ of the control. Successive challenges with caffeine were performed at intervals of 7,10 , and $20 \mathrm{~min}$. Note that the last tension elicited was slightly larger than the first response indicating full recovery of responsiveness. The caffeine was washed out with a saline containing $20 \mathrm{~mm} \mathrm{Mn}$ (arrow). A similar sequence of challenges was made with $\mathrm{Mn}$ always present in the bathing solution (lower row). The recovery of responsiveness was markedly slower; an interval of $40 \mathrm{~min}$ between tensions was not enough to allow the full recovery of responsiveness. $20 \mathrm{~min}$ after removing the $\mathrm{Mn}$ the response to caffeine was larger than before applying Mn.

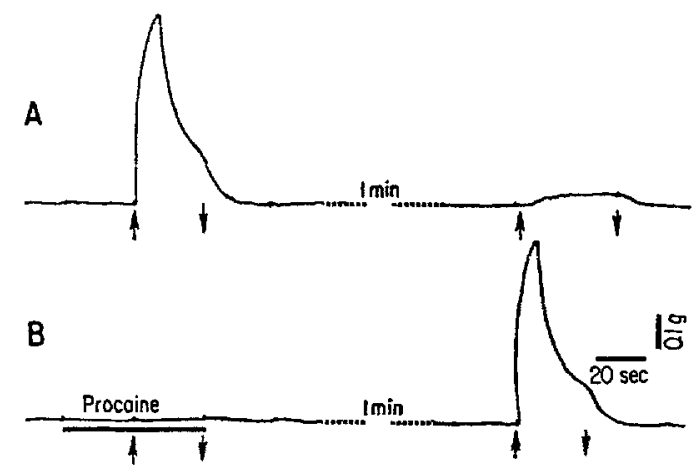

Figure 5. Effect of blocking the initial response to caffeine with procaine. $A$, control series. After the first application of caffeine a second challenge about 2 min later shows the characteristic refractoriness. $B$, the same sequence repeated 20 min later except that procaine $\left(10^{-3} \mathrm{~g} / \mathrm{ml}\right)$ was applied (solid line) and the first challenge with caffeine did not elicit tension. Both drugs were removed and $10 \mathrm{~mm}$ caffeine was again applied 2 min later. Tension similar to that of the control $(A)$ was now obtained. 
$\mathrm{g} / \mathrm{ml}$ ) was applied for $1 \mathrm{~min}$. During the last $30 \mathrm{sec}$ of this caffeine was also applied (arrows). As already described (see accompanying paper) procaine abolishes responsiveness to caffeine and therefore no tension was observed during this challenge. The procaine and caffeine were then removed. After $2.5 \mathrm{~min}$ in the control saline a second challenge with caffeine evoked a tension that was equal to that of the initial challenge in the upper row.

Procaine does not block the entry of caffeine (Bianchi, 1962) while the "release" of Ca from the sarcotubular preparations by caffeine is blocked by procaine (Weber and Herz, 1968). When these results are applied to the living crayfish muscle fiber it would appear that the muscle fiber does not become refractory to a second challenge with caffeine if the release of $\mathrm{Ca}$ by the first challenge is prevented. Thus, the data of Fig. 5 are consistent with

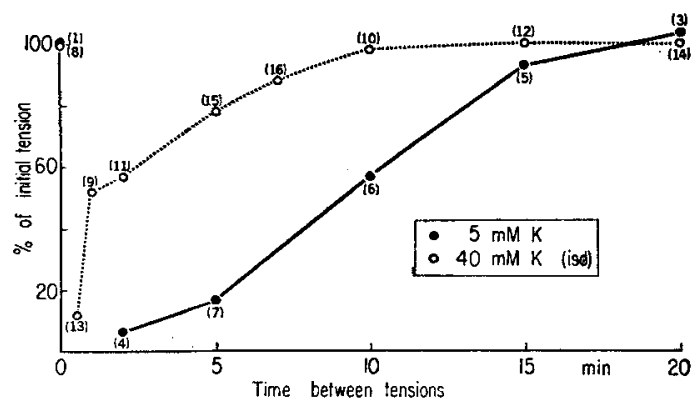

Figure 6. Acceleration of recovery in high $\mathrm{K}_{o}$. Measurements like those described in Fig. 1. Filled circles, control saline; open circles, saline was enriched to $40 \mathrm{~mm} \mathrm{KCl}$ with removal of $\mathrm{Na}$ salt. The control for this series (No. 8) was obtained as described in the text.

the assumption that the period of refractoriness represents the time required to refill the caffeine-sensitive compartment after the latter had been emptied of $\mathrm{Ca}$ by an adequate challenge with caffeine.

\section{B. Acceleration of Recovery of Responsiveness}

RECOVERY IN THE PRESENCE OF HIGH $\mathrm{K}_{o}$ When a fiber is bathed continuously in a medium with elevated $\mathbf{K}_{o}$ recovery after a challenge with caffeine is speeded up considerably (Fig. 6). The solid circles show the recovery (relative to the control response, which is marked No. 1) when the fiber was bathed initially in the standard saline, as described in connection with Fig. 1. The fiber was then exposed to an isosmotic saline containing $40 \mathrm{~mm} \mathrm{~K}$ and allowed to relax from the $\mathrm{K}$-induced contraction. The first subsequent challenge with caffeine results in a potentiated response (see accompanying paper, Fig. 5, and Fig. 10 below). Therefore, the control response for this series of measurements (open circles) was to the next challenge with caffeine made 20 
min after the first. The responses to subsequent challenges are expressed in terms of this control, which is marked No. 8 in Fig. 6. Elevation of $\mathrm{KCl}$ to $40 \mathrm{~mm}$ reduced the recovery time to about half.

EFFECTS OF BRIEF EXPOSURES TO HIGH $\mathbf{K}_{\boldsymbol{o}}$ Recovery of responsiveness to caffeine is also accelerated by brief depolarizations with high $\mathrm{K}_{o}$ (Fig. 7). In this experiment $50 \mathrm{~mm} \mathrm{KCl}$ was applied briefly within $2.5 \mathrm{~min}$ after an initial challenge with caffeine. The $\mathrm{K}$ itself induced tension. 1 min after removing the high $\mathrm{K}_{o}$, or approximately $4 \mathrm{~min}$ after the initial challenge with caffeine, and after the fiber had repolarized to a considerable degree, the caffeine was again applied. It induced a very large and prolonged tension. The excess tension above the control reflects the potentiating effect of high $\mathrm{K}_{0}$. 3 min later another challenge with caffeine, but this time without prior application of $\mathrm{KCl}$, induced negligible tension. The effect of $\mathrm{K}$ on the recovery of responsiveness to caffeine is observed with low concentrations of $\mathrm{K}$ as shown

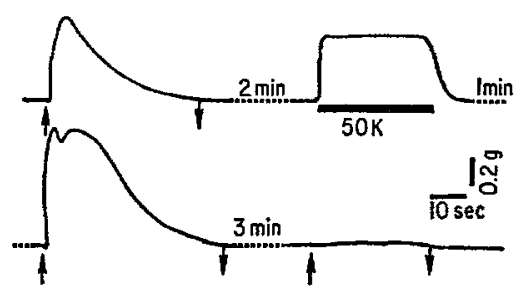

Figure 7. Acceleration of recovery by a brief exposure to high $\mathrm{K}_{0}$. Upper row, the control challenge was followed about $2.5 \mathrm{~min}$ later by brief elevation of $\mathbf{K}_{0}$ to $50 \mathrm{mM}$ (solid line in this and subsequent figures). Another challenge with caffeine $1 \mathrm{~min}$ later (lower row) evoked a more prolonged tension with a larger peak amplitude than in the control. A third challenge $3 \mathrm{~min}$ later elicited almost no tension.

in Fig. 8. In $A$ a control caffeine tension was obtained and after washing out the drug the fiber was bathed for $100 \mathrm{sec}$ in normal saline. At the end of this period caffeine was applied again (second pair of arrows), without evoking tension. 20 min later the procedure was repeated $(B)$, except that after removing the drug a solution containing $15 \mathrm{~mm} \mathrm{KCl}$ was applied for $75 \mathrm{sec}$ (heavy line). This saline was replaced by the normal medium to allow repolarization of the fiber and a test challenge with caffeine was made. Now caffeine elicited a tension about $30 \%$ of the control. The same sequence was performed again, but with a $25 \mathrm{~mm} \mathrm{KCl}$ solution added between the two challenges with caffeine. This concentration of $\mathrm{K}$ itself evoked a small prolonged tension as is evident in the record. After the removal of the high $\mathrm{K}$ the second application of the drug induced a tension $80 \%$ of the control value.

EFFECT OF PRIOR APPLICATIONS OF CAFFEINE ON RESPONSES TO $\mathrm{k}$ The tensions elicited by 50 and $25 \mathrm{~mm} \mathrm{KCl}$ after a caffeine-evoked tension in the 
experiments of Figs. 7 and 8 were considerably smaller than the responses expected in the absence of prior stimulation by caffeine. This is an effect of caffeine as is seen clearly in the records of Fig. 9. In $A$, two successive responses to $50 \mathrm{~mm} \mathrm{~K}$ are shown. In $B$, a response to caffeine preceded the challenge with elevated $\mathrm{K}_{o}$, and the response to the latter was clearly reduced in amplitude. Although not shown in the records, the responses to $\mathrm{K}$ become

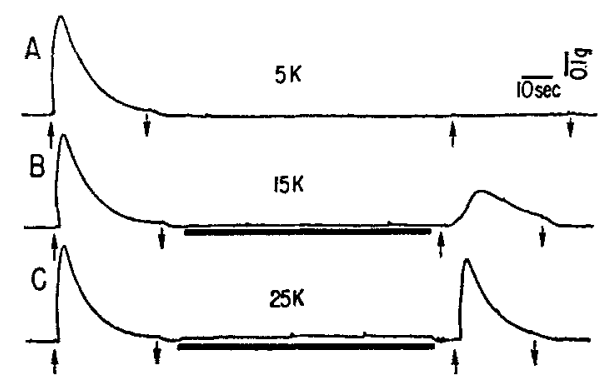

FiguRE 8. Effect of stepwise increase of $\mathrm{K}_{o}$ on the recovery of responsiveness. $A$, control. The first challenge with caffeine induced a tension of about $0.4 \mathrm{~g}$. A second application $100 \mathrm{sec}$ later did not elicit tension. $B, 20 \mathrm{~min}$ later. After the first challenge, a saline containing $15 \mathrm{~mm} \mathrm{~K}$ was applied for about $80 \mathrm{sec}$ (solid line). The high $\mathrm{K}$ saline was then replaced by normal saline. A second challenge with caffeine induced a tension of about $30 \%$ of the control. $C$, a similar sequence $20 \mathrm{~min}$ later except that the fiber was exposed to $25 \mathrm{~mm} \mathrm{~K}$. Note the small tension elicited by the challenge with $\mathrm{K}$. The second challenge with caffeine induced a tension about $80 \%$ of the control.

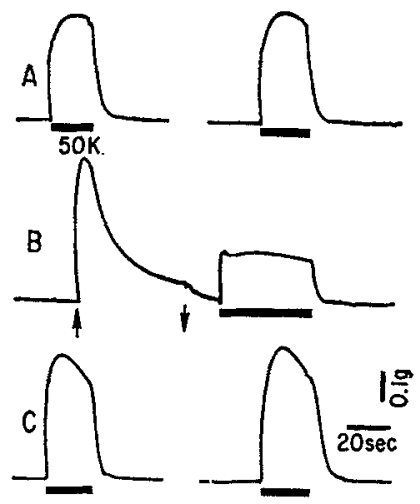

FIGURE 9. Depression of $\mathrm{K}$-induced tension by caffeine. $A$, two responses to $50 \mathrm{mM} \mathrm{K}$ at an interval of $5 \mathrm{~min} . B$, response to $50 \mathrm{~mm} \mathrm{~K} 10 \mathrm{sec}$ after terminating a challenge with $10 \mathrm{mM}$ caffeine was $40 \%$ of those in $A$. $C$, responses to $50 \mathrm{~mm} \mathrm{~K} 5$ and 15 min after $B$.

successively smaller following a sequence of challenges with caffeine. In $C$ are shown the responses to high $\mathrm{K}_{o}$ obtained 5 and $15 \mathrm{~min}$ after the records shown in $B$. The amplitude of these tensions is larger than in the controls $(A)$.

Although applications of high $\mathbf{K}_{o}$ accelerate the recovery of responsiveness to caffeine (Figs. 6-8) the contractions that are evoked by $K$ are themselves 
reduced after a response to caffeine (Figs. 7-9). Thus, the two modes of contractile activation appear to be interdependent, but in a peculiar manner. On the one hand, it appears that the reduction by caffeine of the available store of $\mathrm{Ca}$ in the SR reduces the subsequent response to high $\mathrm{K}$ (Figs. 7-9). This is to be expected if the application of a high $\mathbf{K}$ saline induces tension by liberating $\mathrm{Ca}$ from the SR. On the other hand, the high $\mathrm{K}$ accelerates the replenishment of the $\mathrm{Ca}$ store of the $\mathrm{SR}$, as judged from the acceleration of the recovery of responsiveness to caffeine (Figs. 6-8). Thus, the response to high $\mathrm{K}$ could not have depleted further the caffeine-sensitive, Ca-sequestering system of the SR. The foregoing experiments indicate therefore that exposure

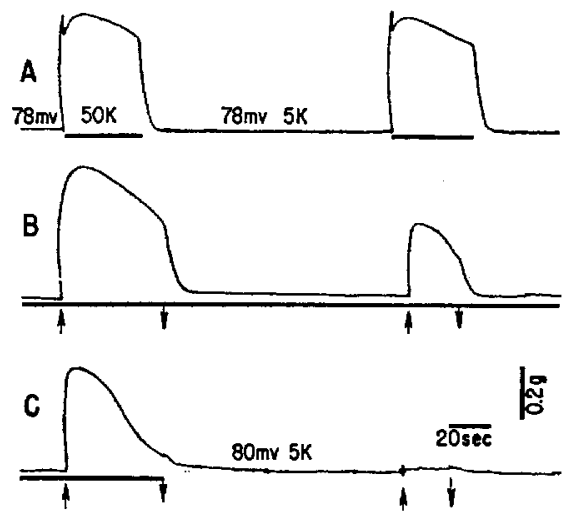

FiguRE 10. Relation of membrane potential to recovery of responsiveness to $\mathrm{K}$ and to caffeine. $A, 2 \mathrm{~min}$ after an initial challenge with $\mathrm{K}$ the repolarized fiber (resting potential $-78 \mathrm{mv}$ ) responded maximally to a second challenge with $50 \mathrm{~mm} \mathrm{~K} . B$, the fiber was equilibrated in the high $\mathrm{K}$ saline, responding to the first challenge by caffeine with a prolonged and larger tension, as described in the text. A second challenge 2 min later, evoked a much smaller response. $C, 20$ min later, with the fiber still in $50 \mathrm{~mm} \mathrm{~K}$. The high $\mathrm{K}$ was removed at the same time as the caffeine and the fiber repolarized to -80 mv. Another challenge with caffeine was now ineffective.

of the fiber to high $\mathrm{K}$ mobilizes a different source of Ca. However, this new supply becomes available to the caffeine-sensitive and temporarily Ca-depleted component as is indicated by the accelerated recovery of responsiveness to caffeine.

DIFFERENGES BETWEEN REGOVERY OF RESPONSIVENESS TO K AND TO CAFFEINE Data on this score serve to emphasize that the two activators of contraction operate upon different systems for the release of $\mathrm{Ca}$. The muscle fiber of Fig. 10 had a resting potential of $-78 \mathrm{mv}$. Its response to $50 \mathrm{~mm} \mathrm{~K}$ is shown at the beginning of $A$. The fiber was returned to the control saline for about $2 \mathrm{~min}$ and had repolarized fully during this time. A second challenge with $50 \mathrm{~mm} \mathrm{~K}$ again evoked a large response. The preparation was then 
bathed continuously in $50 \mathrm{~mm} \mathrm{~K}$. At the time of the records in $B$, made in the presence of the high $\mathrm{K}$ (heavy line), the fiber had relaxed and could not respond again to a further increase in $\mathrm{K}$.

However, as already described in connection with Fig. 6, the first challenge with caffeine elicited an even larger response than did the stimuli with $\mathbf{K}$ in $A$. Furthermore, recovery from refractoriness was also speeded by the continuous depolarization of the fiber and a second challenge with caffeine 2 min after the first elicited a substantial tension. The fiber was then kept in the high $\mathrm{K}$ medium for another $20 \mathrm{~min}$, when a new challenge with caffeine evoked the response seen at the beginning of $C$. Both the caffeine and the $\mathrm{K}$ were washed out and the fiber again repolarized rapidly. The resting potential was $-80 \mathrm{mv} 2 \mathrm{~min}$ later, when a second challenge was made. This time, however, the fiber was refractory to the challenge.

Thus, recovery from depolarization is required for repriming the Ca store that is mobilized by $\mathrm{K}$ (Hodgkin and Horowicz, 1960). Recovery of responsiveness to caffeine presumably also signifies the restoration (or repriming) of a $\mathrm{Ca}$ store that is available to the drug. However, repriming of this store is enhanced by depolarization and is slow when the membrane is repolarized from a previous depolarization. It would appear as if the two Ca stores stand in a reciprocal relation. When one store is depleted by depolarization, replenishment of the other is accelerated. When the K-sensitive store is being replenished by repolarization, the repriming of the caffeine-sensitive store is slow. The relation must be complicated, however, in view of the interdependence that was shown in Fig. 9.

About 10 times more EGTA is required to block the release of "intracellular" Ca by high $\mathrm{K}$ in barnacle muscle fibers than by caffeine (Ashley, 1967). Thus it is likely that in this muscle also $\mathrm{K}$ utilizes a different source of $\mathrm{Ca}$ than does caffeine. The responses of crayfish fibers to $\mathrm{Ca}$ injection and to caffeine are also affected differently by various agents, including EGTA (Brandt and Grundfest, 1968; Reuben et al., 1969, 1970 and unpublished data).

\section{G. Role of the Membrane Potential in Accelerating Recovery of Responsiveness}

It might be supposed from the foregoing data that the depolarization of the muscle fiber which is involved in $\mathrm{K}$ contractures itself accelerates the repriming of the caffeine-sensitive Ca store. This is not the case, since muscle fibers can be depolarized without accelerating recovery of responsiveness to caffeine.

RECOVERY IN A LOW Ca MEdIUM Fig. $11 A$ shows a control response in which a challenge with $50 \mathrm{~mm} \mathrm{~K}$ was applied immediately after a challenge with caffeine. When the $\mathrm{K}$ was washed out a second challenge with caffeine 
made only 2 min after the first evoked a very large response. For the records of Fig. $11 \mathrm{~A}$ the fiber had been equilibrated in the $\mathrm{Cl}$-free control saline. The bathing medium was now changed for a Cl-free saline containing only $3 \mathrm{~mm}$ $\mathrm{Ca}$ and the muscle fiber was equilibrated in this medium for $2 \mathrm{hr}$ prior to the sequence shown in $B$. The resting potential is not affected by exposure to low $\mathrm{Ca}$ in a $\mathrm{Cl}$-free saline, but when the fiber is depolarized on exposure to high $\mathrm{K}$ there is no contractile response (Reuben et al., 1967 a). An application of caffeine, however, evoked the response shown at the beginning of the record. The fiber was then depolarized by applying $50 \mathrm{~mm} \mathrm{~K}$ (solid line) without causing a contraction. A second challenge with caffeine (second set of arrows) evoked a tension only about $5 \%$ of the control. Thus, while the

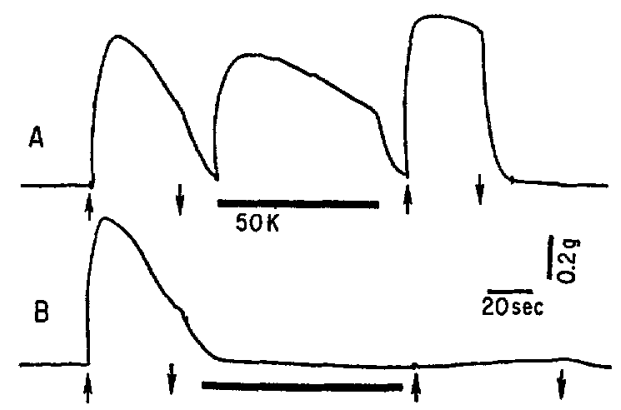

Figure 11. Effect of low Ca on repriming of responsiveness to caffeine by high $\mathbf{K}_{0}$. $A$, fiber in Cl-free (propionate) control saline. Responsiveness to a second challenge with caffeine restored by an intervening challenge with $\mathrm{K} . B$, fiber soaked for $2 \mathrm{hr}$ in the $\mathrm{Cl}$-free saline, but with $\mathrm{Ca}$ reduced to $3 \mathrm{~mm}$. The response to the initial challenge with caffeine was essentially unaffected, but $50 \mathrm{~mm} \mathrm{~K}$ did not evoke tension. Only a small response was evoked by a second challenge with caffeine.

fiber had depolarized when $\mathbf{K}_{\boldsymbol{o}}$ was raised, there was no acceleration of recovery to caffeine.

RECOVERy IN THE PResence of mn The foregoing experiment did not rule out, however, the possibility that the hypothetical intracellular Ca store which is responsive to depolarization, but not to caffeine, may have been selectively affected by the low $\mathrm{Ca}_{0}$. Experiments like that shown in Fig. 12 were therefore designed to test this possibility, by taking advantage of the effect of $\mathrm{Mn}$ in blocking the contractile activation of crayfish muscle fibers by $\mathrm{K}$. The fiber in the experiment of Fig. 12 was bathed in the standard Cl-free saline which contains $13.5 \mathrm{~mm}$ Ca. The sequences of records $A$ and $B$ are controls, without repriming by $\mathrm{K}(A)$ and with responsiveness accelerated by $40 \mathrm{~mm} \mathrm{~K}(B) .20 \mathrm{~min}$ after $B$ the fiber was exposed to another challenge with caffeine $(C)$ but in the presence of $20 \mathrm{~mm}$ Mn (broken line). Although $\mathrm{Mn}$ itself slows the recovery of responsiveness to caffeine (Fig. 4), the presence 
of $\mathrm{Mn}$ does not affect the tension evoked by caffeine and the response to the first challenge with caffeine in Fig. $12 C$ was similar to the initial control responses in $A$ and $B$. The caffeine was removed and the fiber was depolarized with $40 \mathrm{~mm} \mathrm{~K}$ while retaining the $20 \mathrm{~mm} \mathrm{Mn}$. There was no response and a second challenge with caffeine also did not elicit a response.

It will be recalled that the experiment of Fig. 12 was performed in the presence of the full complement of $\mathrm{Ca}_{o}$. It would therefore be necessary to postulate that the hypothetical $\mathrm{K}$-sensitive intracellular Ca store was rendered unresponsive to depolarization because of the presence of $\mathbf{M n}$ in the bathing medium. This is unlikely for two reasons. Mn probably does not move across the cell membrane of crayfish muscle fibers, since the Ca spikes of these

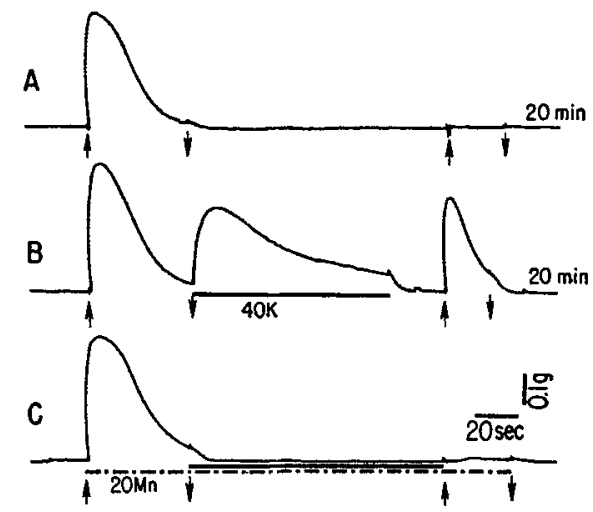

FiguRE 12. Effect of $\mathrm{Mn}$ on repriming by high $\mathrm{K}$. $A$, control series showing that the fiber was refractory to a second challenge with caffeine made 2 min after the first. $B$, a similar sequence repeated $20 \mathrm{~min}$ later but with an intervening challenge with $40 \mathrm{~mm} \mathrm{~K}$. C, $20 \mathrm{~min}$ later $\mathrm{Mn}(20 \mathrm{~mm}$ ) was introduced with the caffeine and was present in the bath thereafter as shown by the broken line. The Mn blocked a response to $40 \mathrm{~mm} \mathrm{~K}$ and a subsequent challenge with caffeine was ineffective.

fibers are blocked by Mn (Takeda, 1967). Furthermore, Mn itself can activate the contractile machinery when injected into an intact fiber or when applied to the skinned fiber preparation (Reuben et al., unpublished data), but it does not cause contraction when applied externally to the intact fiber. Thus, it seems reasonable to assume that the effect of Mn observed in Fig. 12 is due to its block of $\mathrm{Ca}$ entry into the muscle fiber.

In summary, it is evident that depolarization alone is insufficient to accelerate recovery of responsiveness to caffeine. Depolarization is ineffective when the application of $\mathbf{K}$ fails to evoke a contraction. This failure is observed when there is insufficient $\mathrm{Ca}$ in the external medium (Fig. 11) or when the entry of $\mathrm{Ca}$ from the exterior is blocked by Mn (Fig. 12). Thus, it seems that the acceleration by high $\mathrm{K}$ of recovery of responsiveness to caffeine must be 
related to the availability of $\mathrm{Ca}$ in the external medium. In other words, the depolarization that is induced by $\mathrm{K}$ "mobilizes" Ca from the bathing medium and the Ca entering from this external source becomes available to reprime the responsiveness of the $\mathrm{SR}$ to caffeine.

REPRIMING WITH INTRACELLULARLY APPLIED GURRENTS If the above conclusion is valid it would seem likely that depolarization of the muscle fiber by an electric current might also accelerate the recovery of responsiveness to caffeine. This was confirmed by experiments like that shown in Fig. 13. The records of the upper row $(A)$ show a control series with the fiber equilibrated in the standard $\mathrm{Cl}$ saline. After the initial response to caffeine, a second challenge delivered about 2 min later evoked only a small response. The fiber was now rested for $20 \mathrm{~min}$ before beginning the sequence shown in $B$. The re-

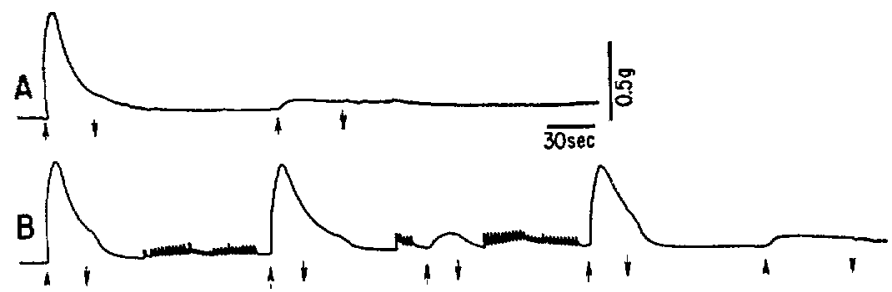

Figure 13. Repriming by intracellularly applied depolarizing currents. $A$, control sequence showing refractoriness to a second challenge with caffeine about 2 min after the first challenge. $B, 20$ min later. After the initial challenge pulses of outward current (ca. $2 \times 10^{-7} \mathrm{amp}$ ) were applied at $0.5 / \mathrm{sec}$. Each evoked a small contraction. Current was reduced midway in the train to prevent a large summated contraction. A second challenge with caffeine about 2 min after the first now evoked large tensions. Less recovery followed a shorter train of pulses. Recovery was large again after a third and longer train. In the absence of prior stimulation by current recovery was again small.

sponse to the initial challenge with caffeine was similar to the corresponding response in $A$. Pulses of depolarizing current (ca. $2 \times 10^{-7} \mathrm{amp}$ ) were then applied intracellularly at a frequency of about $0.5 / \mathrm{sec}$. These currents cause depolarizations ranging between 20 and $40 \mathrm{mv}$ in crayfish muscle fibers and are sufficient to evoke small tensions. The tensions evoked by these pulses were beginning to summate and for the next $30-45 \mathrm{sec}$ the current was reduced somewhat. The stimuli were then stopped and the fiber was challenged by caffeine. The response was a nearly maximal tension. A briefer train of pulses preceded another challenge with caffeine. Although this challenge was made only about $1.5 \mathrm{~min}$ after the previous one considerable tension was evoked. Subsequently another prolonged train of pulses again enhanced a response to caffeine. About $1.5 \mathrm{~min}$ after this nearly maximal tension the fiber was challenged once more, but without intervening electrical stimulation. This last response was of about the same magnitude as the second response in $A$. 
The depolarizing currents evoked small contractions and, thus, presumably they mobilized $\mathrm{Ca}$ within the myoplasm. If the current released the $\mathrm{Ca}$ from the same source as does caffeine, a slowing of the recovery after an application of a train of pulses would be anticipated. On the contrary, the recovery was accelerated, just as it was when the contraction of the fiber was activated by $\mathrm{K}$. The recovery as well as the contractile activity induced by the depolarizing currents was abolished in the presence of Mn. Hyperpolarizing (inward) currents did not accelerate recovery of responsiveness even when the current was increased threefold over that used to depolarize the fiber.

ROLE OF Cl IN REPRIMING Recovery of responsiveness is affected by a number of maneuvers in which the external anion ( $\mathrm{Cl}$ in the standard saline) is altered. Details of these studies will be reported elsewhere. At the present time we shall describe only one condition in which recovery of responsiveness

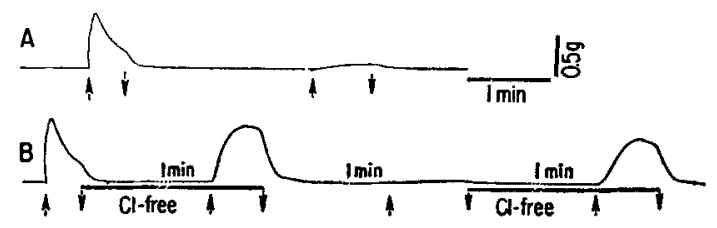

Figure 14. Repriming of responsiveness to caffeine by removal of $\mathrm{Cl}$. $A$, control sequence. The second challenge with caffeine was made after about $2 \mathrm{~min}$. $B$, after an interval of $20 \mathrm{~min}$. When the first application of caffeine was removed a Cl-free (propionate) saline was introduced (broken line). The second challenge with caffeine evoked a large but slowly rising tension. The $\mathrm{Cl}$ saline was replaced and a third challenge with caffeine found the fiber unresponsive, but on removing $\mathrm{Cl}$ again, a fourth challenge again evoked tension.

to caffeine is accelerated, by substitution of the impermeant anion propionate for the normal Cl. Row $A$ in Fig. 14 shows a control sequence with the fiber in the $\mathrm{Cl}$ saline. $2 \mathrm{~min}$ after the initial test challenge, a second challenge with caffeine elicited only a small response. The fiber was equilibrated in the $\mathrm{Cl}$ saline for another $20 \mathrm{~min}$ thereafter. The initial challenge $(B$, first set of arrows) elicited essentially the same response as did the corresponding challenge in $A$. Now, however, the caffeine was washed out with the $\mathrm{Cl}$-free saline, to which the fiber was exposed for about $3 \mathrm{~min}$ (heavy line). After $2 \mathrm{~min}$ in this saline the fiber was again challenged with caffeine (second set of arrows) and this now induced a large, though slowly rising and prolonged response. The caffeine was washed out this time with the $\mathrm{Cl}$ saline. A third challenge with caffeine elicited very little tension. However, on reexposing the fiber to the Cl-free saline (second heavy line) recovery was again accelerated (fourth set of arrows).

The foregoing experiment does not add to the analysis of the nature of the repriming mechanism. It is included at this time, however, to emphasize that 
the processes involved must be quite complex. $\mathrm{Cl}$ and other anions of the lyotropic series induce contraction in the skinned fiber preparations of frog (Costantin et al., 1965) and crayfish (Reuben et al., 1967 b). These anions and particularly the larger ones (e.g. SCN) greatly potentiate the contractions evoked in crayfish muscle fibers by intracellular injections of $\mathrm{Ca}$ (Reuben et al., 1969). Nevertheless, it is the removal of $\mathrm{Cl}_{o}$ that accelerates the recovery of responsiveness to caffeine. This experimental maneuver induces a loss of intracellular Cl, but only of a mobile fraction (Dunham et al., 1964; Dunham and Gainer, 1968). The replacement of $\mathrm{Cl}$ with an impermeant anion induces a brief depolarization, which though small (Girardier et al., 1963), is probably the initiator of the repriming of responsiveness to caffeine.

\section{I S G U S I O N}

When the application of caffeine induces a contraction in crayfish muscle fibers - and only then (Fig. 5) - the fibers become refractory to a second challenge with caffeine. Full recovery requires some 15-20 min. Lüttgau and Oetliker (1968, p. 54) state that in the frog intervals as long as $40 \mathrm{~min}$ between applications are required to yield satisfactorily reproducible results even with low concentrations of caffeine. This suggests the possibility that similar refractoriness may also occur in frog muscle, but we know of no systematic study analogous to the present work on crayfish fibers. Recovery of responsiveness to caffeine can be accelerated by high $\mathrm{K}$ and by intracellularly applied outward currents, but only when the depolarizations that these stimuli evoke also elicit contractions.

There is general agreement (see recent reviews by Sandow, 1965; Weber, 1966; Bianchi, 1968; Ebashi and Endo, 1968) that the contraction of muscle fibers whether evoked by electrical stimulation, including the action potential and depolarization by $\mathbf{K}$, or by various chemical agents, including caffeine, is induced by an increase in intracellular free Ca. Most of the workers hold that $\mathrm{Ca}$ is released from an intracellular store, which by sequestering the $\mathrm{Ca}$ in the resting fibers, maintains the myoplasmic concentration below that needed for contraction. This store is generally believed to be located in the SR.

The data presented in this series of papers demonstrate that at least two different sources of Ca participate in the activation of contraction in crayfish muscle fibers. One source is a caffeine-sensitive intracellular store, very likely in the SR; the other is dependent on the presence of $\mathrm{Ca}$ in the bathing medium and upon the permeability of the cell membrane to Ca. Caffeine acts principally to release $\mathrm{Ca}$ from the intracellular store and once the $\mathrm{Ca}$ is emptied from that source the store is replenished only slowly. Electrical stimulation, i.e. depolarization, whether with $\mathrm{K}$ or with intracellularly applied current, mobilizes the other, presumably superficial source, and apparently 
also induces a rapid influx of $\mathrm{Ca}$ from the exterior. Once this second source of $\mathrm{Ca}$ is made available to the contractile proteins, it can be utilized also to replenish the store that had been exhausted by caffeine.

The Intracellular Store of $\mathrm{Ca}$ Our assumption that the uptake of $\mathrm{Ca}$ is inhibited by caffeine and the release of $\mathrm{Ca}$ is inhibited by procaine is consistent with the findings of Weber and Herz (1968) on frog and rabbit sarcotubular preparations and it is also consistent with the physiological data. The SR of crayfish muscle can sequester Ca very strongly (Grundfest, 1966, Fig. 25). Thus, the SR is probably the major intracellular Ca store, as it is in vertebrate muscle (Hasselbach and Makinose, 1961; Costantin et al., 1965; Winegrad, 1968; Weber and Herz, 1968). We do not have direct evidence, however, that it is the SR component which is involved, as proposed, in contractile activation by caffeine.

The Refractoriness after a Challenge with Caffeine Several explanations could be given for the slow recovery of responsiveness that follows a contraction evoked by caffeine (Fig. 1). It could be argued that the refractoriness after the caffeine of the first challenge had been removed, is due to a slow reversal of the inhibitory effect of caffeine on the sequestration of $\mathrm{Ca}$ by the $\mathrm{SR}$, or that a contraction evoked with caffeine is followed by an exhaustion of the energy source used for the contraction. However, these explanations are inconsistent with the immediate recovery of responsiveness to caffeine induced by treatments such as elevated $\mathrm{K}$ and depolarizing currents.

Since the response to caffeine is transient (see accompanying paper), a consequence of the mechanism postulated for the action of caffeine in evoking a contraction is that the Ca store should be temporarily reduced or even depleted after a caffeine tension. Thus a more likely explanation for the refractoriness is that it signals a depletion of the Ca store of the SR. This point of view is supported by the finding that there is refractoriness to a second application of caffeine only when the first challenge had elicited tension, as is shown in the experiment with procaine (Fig. 5). Furthermore, the degree to which the fiber is made refractory to a second challenge with caffeine depends upon the duration of the action of the first challenge (Fig. 2). This is consistent with the proposed schema. Caffeine enters and leaves the fiber rapidly (Bianchi, 1962) and when the duration of caffeine action is short the reduction of the Ca store of the SR and its sequestration by a caffeine-insensitive compartment must be minimal. However, the major part of the depletion process must have occurred within $6 \mathrm{sec}$ (Fig. 2). The speed of restoration of the $\mathrm{Ca}$ store must be related to the availability of $\mathrm{Ca}$ in the myoplasm and to the rate of $\mathrm{Ca}$ accumulation by the SR. Since it has been shown (Ohnishi and Ebashi, 1964) that the SR sequesters Ca at a very high rate, the limiting step would seem to be the availability of Ca. 
The recovery of responsiveness has the same time course after challenges with $40 \mathrm{~mm}$ caffeine as with $10 \mathrm{~mm}$ caffeine, although the amplitudes of the respective responses are larger with the higher concentrations of caffeine. However, in both cases the tension-time integrals are very similar (see Fig. 2 of accompanying paper). This might indicate that the total amount of $\mathrm{Ca}$ released by 10 and $40 \mathrm{~mm}$ of caffeine is the same although the time courses of the effects are different. It was not possible to compare the recovery of responsiveness for tensions with different tension-time integrals, as for example those evoked by 2 and $10 \mathrm{~mm}$ caffeine, since concentrations below $5 \mathrm{~mm}$ do not produce a uniform activation of the fiber (see accompanying paper). Assuming that there is a second Ca-sequestering system (see accompanying paper) the site (or sites) of the caffeine-insensitive mechanism, the kinetics of the sequestration, as well as its competitive relation with the $\mathrm{SR}$, when the latter can take up Ca again on removal of caffeine, are all unknown. In crayfish the mitochondria are concentrated at the periphery of the muscle fibers (Brandt et al., 1965). They, as well perhaps as a second component of the SR (Weber and Herz, 1968), might act as the second, caffeine-insensitive system. It has been suggested on the basis of radioautography (Winegrad, 1968) that the terminal sacs of the SR are the sites of Ca release, while the Ca uptake is effected by the longitudinal regions of the SR.

Another possible element in the second sequestering system may be the cell membrane itself. An active transport (pump) mechanism for $\mathrm{Ca}$ is probably essential to maintain a low intracellular concentration of $\mathrm{Ca}$ in the face of a diffusional gradient of about $10^{5}$ (Weber, 1966; Bianchi, 1968; and the accompanying paper). Experiments like that illustrated in Fig. 7 of the accompanying paper also indicate that the active extrusion of $\mathrm{Ca}$ may play a large role.

There is the further likelihood, too, that the release (and uptake) of $\mathrm{Ca}$ by the SR and/or other intracellular systems are nonlinear processes. Responses involving feedback have been observed in the experiments with injections of $\mathrm{Ca}$ into intact fibers (Reuben et al., 1969, 1970). The slow rates of tension development of the small responses observed during the refractory period (e.g., Figs. 1, 4, and 8) are analogous to the slowly developing tension of the plateau phase of the responses to the injection of $\mathrm{Ca}$, when the early component of the increase in tension is abolished by procaine.

The Role of External Ca Hill (1948) offered a convincing argument against the suggestion by Heilbrunn and Wiercinski (1947) that Ca release "from the surface or outer region of the cell ... enters the cell and produces the (contractile) response." Since then, the role of external Ca in contraction has been discounted (for discussion cf. Sandow, 1965) despite evidence that during contractile activity there is an enhancement of the Ca entry (Bianchi 
and Shanes, 1959; Lorković, 1962; Edwards and Lorković, 1967; Gainer, 1968). The present work offers a new type of evidence for the participation of extracellular $\mathrm{Ca}$ in the contractile activity. Depolarizations that are induced by $\mathrm{K}$ or by applied currents and which produce contractile activity mobilize a source of $\mathrm{Ca}$ that is not that of the caffeine-sensitive Ca store of the SR and this supply of $\mathrm{Ca}$ becomes available to replenish the latter system.

Removal of $\mathrm{Ca}$ slows the recovery to such an extent that the muscle fiber becomes unresponsive to repeated challenges with caffeine (see the accompanying paper). This finding suggested that maintenance of the $\mathrm{Ca}$ store for chemical activation does in fact depend upon a contribution of $\mathrm{Ca}$ from the medium. This is borne out by the experiments with Mn (Fig. 4). The latter blocks the electrogenesis ascribed to the entry of $\mathrm{Ca}$ into barnacle and heart muscle (Hagiwara and Nakajima, 1966; Rougier et al., 1969) and it also depresses procaine-induced Ca spikes of crayfish muscle (Takeda, 1967). The marked slowing of recovery of responsiveness to caffeine thus is in keeping with the possibility that $\mathrm{Mn}$ had reduced the resupplying of the Ca store of the SR by reducing the entry of Ca from the bath.

Increase of $\mathrm{Ca}_{o}$ to $50 \mathrm{~mm}$ had no measurable effect on the recovery of responsiveness (Fig. 3). This finding may be regarded as an indication that even in the presence of an almost fourfold increase in $\mathrm{Ca}_{0}$ the rate of influx of $\mathrm{Ca}$ in the absence of caffeine was still small. However, on raising $\mathrm{Ca}_{o}$ the responses to caffeine are considerably augmented. This finding indicates that the $\mathrm{Ca}$ made available to the myoplasm from the external medium is augmented during a caffeine contraction.

Acceleration of Recovery The acceleration of recovery of responsiveness to caffeine by $\mathbf{K}$ or by intracellularly applied currents is somewhat analogous to the speeding of the recovery of responsiveness to $\mathrm{K}$-induced contractures by repolarization, which has been termed repriming of responsiveness (Hodgkin and Horowicz, 1960). An essential aspect of difference, of course, is the fact that accelerated repriming of the chemically evoked responsiveness is not induced by repolarization (Fig. 10).

Repolarization also reprimes the responsiveness to $\mathrm{K}$ of crayfish muscle fibers (Fig. 10; Zachar and Zacharova, 1966; Reuben et al., 1967 a). Zachar and Zacharova (1966), who studied Astacus, reported that the repriming is very slow if the initial challenge with $\mathrm{K}$ is maintained until the contracture has subsided. This was not observed in the studies of this laboratory employing Procambarus or Orconectes. However, the slowed recovery in Astacus was associated with slow repolarization (Zachar and Zacharova, 1966, Fig. 11). In the present work repolarization after a very prolonged (>20 min) exposure of the fiber to high $\mathrm{K}$ was about as rapid (Fig. $10 \mathrm{C}$ ) as it was after a shorter exposure (Fig. $10 \mathrm{~A}$ ). 
It appears as though the requirement for accelerated repriming of responsiveness to caffeine is to establish conditions that permit entry of $\mathrm{Ca}$ into the fiber. An increase in $\mathrm{K}_{\mathrm{o}}$ is known to meet the above requirements since this enhances the movement of $\mathrm{Ca}$ across the surface membrane of muscles (Bianchi and Shanes, 1959; Lorković, 1962; Edwards and Lorković, 1967; Gainer, 1968). It is noteworthy that even brief exposures of the fiber to high $\mathrm{K}$ (Fig. 7) are adequate to induce the acceleration. Furthermore, after a brief exposure to $\mathrm{K}$ had induced tension and depolarization, the responsiveness to caffeine remained high, although the membrane potential was again back to the resting level (Figs. 7 and 8). Presumably, the application of $\mathrm{K}$ initiated an influx of $\mathrm{Ca}$ large enough to reprime the SR store and, once accumulated at this site, the $\mathrm{Ca}$ remained available until the next challenge.

Although our data indicate that the entry of $\mathrm{Ca}$ is an important and perhaps the causative element in accelerating recovery to caffeine, it should be borne in mind that the full course of the acceleration is not necessarily dependent solely on this supply of Ca. Tension evoked by iniection of Ca clearly exhibits components that can be modified independently by a number of environmental factors (Reuben et al., 1969, 1970 and unpublished data). The regenerative effect appears to be due to an entry of extracellular Ca over and above that which is injected. Thus, it is likely that there is a complex interrelation between the intracellular Ca-sequestering system (or systems if there should be more than one) and the supplying of $\mathrm{Ca}$ from the exterior. The electrical mode of contractile activation appears to show some degree of dependence on the $\mathrm{Ca}$ store of the $\mathrm{SR}$ since $\mathrm{K}$-induced tension immediately following a caffeine-induced response is transiently diminished (Fig. 9). However, caffeine elicits maximal tensions immediately following a maximal $\mathrm{K}$-induced contraction and this signifies that the $\mathrm{SR}$ store available to caffeine is not depleted by the high $\mathbf{K}_{0}$. Furthermore, if activation by high $\mathbf{K}$ were to mobilize a significant amount of $\mathrm{Ca}$ from the $\mathrm{SR}$ it would be unlikely that the increased $K$ could accelerate the recovery of responsiveness to caffeine. In fact, the acceleration is proportional to the amplitude of the $\mathrm{K}$-induced tensions (Fig. 8).

Excitation of the muscle fibers with intracellularly applied currents also accelerates recovery of responsiveness to caffeine (Fig. 13). Noteworthy is the fact that the depolarizations were brief, and repeated only at 2 sec intervals. Furthermore, longer trains of pulses speeded recovery more. This suggests an effect of the quantity of charge, which in this case would be the influx of Ca that is induced by each depolarization.

Redistribution of intracellular Cl that results from the withdrawal or rereplacement of $\mathrm{Cl}$ by an impermeant anion is associated with rather small changes in membrane potential (Reuben et al., 1964; cf. Figs. 3-5 in Grundfest, 1962). Tension frequently accompanies the transient depolarization, in- 
dicating that the withdrawal of $\mathrm{Cl}$ mobilizes a source of $\mathrm{Ca}$ that becomes available for activating the contractile system. Thus, the repriming of responsiveness to caffeine upon withdrawal of $\mathrm{Cl}$ (Fig. 14) may result from effects that are similar to those induced by depolarizing currents or by high $\mathrm{K}_{0}$.

The kinetics of the Ca-dependent events in muscle may be further complicated by the existence of several other systems controlling the interaction of Ca with the contractile mechanism. Daniel (1965) has summarized the views of many workers who favor "the probable existence of two types of sites at which $\mathrm{Ca}$ and other divalent ions interact with the muscle membrane and endoplasmic reticulum of all types of muscle" (p. 295). Thus, the entry of $\mathrm{Ca}$ that is induced by depolarization may interact with at least two sequestering mechanisms and if so, the kinetics of various Ca-dependent events in muscle will be regulated by the existence of at least three Ca-controlling systems, the cell membrane and the several intracellular components.

In summary, and assuming that $\mathrm{Ca}$ is the primary activator of the contractile system of crayfish muscle fibers, the foregoing work has shown that tension which is induced by caffeine is initiated by mobilization of $\mathrm{Ca}$ from a source that is distinct, at least to a very large degree, from the source (or sources) available for electrical modes of activation (current, high $\mathrm{K}_{o}$, withdrawal of $\mathrm{Cl})$. The distinction is evidenced $(a)$ by the nature and degrees of interactions between the chemically and electrically evoked contractions and (b) by the different modes of promoting the recovery of responsiveness (repriming) to a given stimulus.

Work in this laboratory is supported in part by funds from the Muscular Dystrophy Associations of America, Inc.; by Public Health Service Research Grant (NB 03728, NB 03270) and Training Grant (NB 05328) from the National Institute of Neurological Diseases and Stroke; and from the National Science Foundation (GB 6988X).

Dr. Chiarandini was an International Fellow of the U. S. Public Health Service. Dr. Reuben holds a Career Development Award (NB 21861) from the National Institutes of Health.

Received for publication 11 September 1969.

\section{RE F E RE N C ES}

Ashley, C. C. 1967. The role of cell calcium in the contraction of single cannulated muscle fibers. Amer. Zool. 7: 647 .

BiAnch, C. P. 1962. Kinetics of radiocaffeine uptake and release in frog sartorius. $J$. Pharmacol. Exp. Ther. 138: 41.

BianchI, C. P. 1968. Cell Calcium. Butterworth \& Co. (Publishers), Ltd., London.

Bianch, C. P., and A. M. Shanes. 1959. Ca influx in skeletal muscle at rest, during activity, and during potassium contracture. J. Gen. Physiol. 42: 803.

Brandt, P. W., J. P. Reuben, L. Girardier, and H. Grundfest. 1965. Correlated morphological and physiological studies on isolated single muscle fibers. I. Fine structure of the crayfish muscle fiber. J. Cell Biol. 25: 233.

Costantin, L. L., C. Franzini-Armstrong, and R. J. Podolsky. 1965. Localization of calcium-accumulating structures in striated muscle fibers. Science (Washington). 147: 158. 
Daniel, E. E. 1965. Attempted synthesis of data regarding divalent ions in muscle function. In Muscle. W. M. Paul, E. E. Daniel, C. M. Kay, and G. Monckton, editors. Pergamon Press, Oxford. 295.

Dunham, P. B., and H. Gainer. 1968. The distribution of inorganic ions in lobster muscle. Biochim. Biophys. Acta 150: 488.

Dunham, P. B., J. P. Reuben, P. W. Brandt, and H. Grundfest. 1964. The distribution of chloride in crayfish muscle fibers. Society of General Physiologists, Woods Hole Meeting. (Abstr.).

Ebashi, S., and M. Endo. 1968. Calcium ion and muscle contraction. Progr. Biophys. Mol. Biol. 18: 125.

EDWARDS, G., and H. LoRković. 1967. The roles of calcium in excitation-contraction coupling in various muscles of the frog, mouse and barnacle. Amer. Zool. 7:615.

GAINER, H. 1968. The role of calcium in excitation-contraction coupling of lobster muscle. J. Gen. Physiol. 52: 88.

Girardier, L., J. P. Reuben, P. W. Brandt, and H. Grundfest. 1963. Evidence for anionpermselective membrane in crayfish muscle fibers and its possible role in excitation-contraction coupling. J. Gen. Physiol. 47: 189.

GRUND Fest, H. 1962. Ionic transport across neural and non-neural membranes. In Properties of Membrane and Diseases of the Nervous System. M. Yahr, editor. Springer Publishing Co., Inc., New York. 71.

GrundFEst, H. 1966. Heterogeneity of excitable membrane: electrophysiological and pharmacological evidence and some consequences. Ann. N. Y. Acad. Sci. 137:901.

Hagrwara, S., and S. Nakajtma. 1966. Differences in $\mathrm{Na}$ and $\mathrm{Ca}$ spikes as examined by application of tetrodotoxin, procaine, and manganese ions. J. Gen. Physiol. 49: 793.

Hasselbach, W., and M. Makinose. 1961. Die Calciumpumpe der "Erschlaffungsgrana" des Muskels und ihre Abhängigkeit von der ATP-Spaltung. Biochem. Z. 333: 518.

Heilbrunn, L. V., and F. J. Wiercinski. 1947. The action of various cations on muscle protoplasm. J. Cell. Comp. Physiol. 29: 15.

Heistracher, P., and C. C. Hunt. 1969. Contractile repriming in snake twitch muscle fibres. J. Physiol. (London). 201: 613.

HiLl, A. V. 1948. On the time required for diffusion and its relation to processes in muscle. Proc. Roy. Soc. Ser. B. Biol. Sc. 135: 446.

Hodgkin, A. L., and Horowicz, P. 1959. The influence of potassium and chloride ions on the membrane potential of single muscle fibres. J. Physiol. (London). 148: 127.

Hodgkin, A. L., and Horowicz, P. 1960. Potassium contractures in single muscle fibres. $J$. Physiol. (London). 153: 386.

LoRković, H. 1962. Potassium contracture and calcium influx in the frog's skeletal muscle. Amer. J. Physiol. 202: 440.

Lütrgau, H. G., and M. Oetliker. 1968. The action of caffeine on the activation of the contractile mechanism in striated muscle fibres. J. Physiol. (London). 194: 51.

OhNishi, T., and S. EBAshI. 1964. The velocity of calcium binding of isolated sarcoplasmic reticulum. J. Biochem. 55: 599.

ReUben, J. P., P. W. BRandt, H. Garcia, and H. Grundfest. 1967 a. Excitation-contraction coupling in crayfish. Amer. Zool. 7:623.

Reuben, J. P., P. W. Brandt, and H. Grundfest. $1967 b$. Tension evoked in skinned crayfish muscle fibers by anions, $\mathrm{pH}$, and drugs. J. Gen. Physiol. 50: 2501.

Reuben, J. P., P. W. Brandt, G. M. Katz, and H. Grundfest. 1970 a. Augmentation of responses to calcium injections by agents which reduce calcium sequestration. $J$. Gen. Physiol. 55: 140.

Reuben, J. P., L. Girardier, and H. Grundfest. 1964. Water transfer and cell structure in isolated crayfish muscle fibers. J. Gen. Physiol. 47: 1141.

Reuben, J. P., G. M. Katz, and M. Berman. 1969. Two phases of contractile activation induced by $\mathrm{Ca}$ injections in crayfish muscle fibers. Fed. Proc. 28: 711.

Rougier, O., G. Vassort, D. Garnier, Y. M. Garcouil, and E. Coraboeuf. 1969. Existence 
and role of a slow inward current during the frog atrial action potential. Arch. gesamte Physiol. Menschen Tiere (Pfluegers). 308: 91.

Sandow, A. 1965. Excitation-contraction coupling in skeletal muscle. Pharmacol. Rev. 17: 265.

TAKedA, K. 1967. Permeability changes associated with the action potential in procainetreated crayfish abdominal muscle fibers. J. Gen. Physiol. 50: 1049.

WEBER, A. 1966. Energized calcium transport and relaxing factors. In Gurrent Topics in Bioenergetics. D. R. Sanadi, editor. Academic Press, Inc., New York. 203.

Weber, A., and R. Herz. 1968 The relationship between caffeine contracture of intact muscle and the effect of caffeine on reticulum. J. Gen. Physiol. 52: 750.

WinEGRAD, S. 1968. Intracellular calcium movements of frog skeletal muscle during recovery from tetanus. J. Gen. Physiol. 51: 65.

Zachar, J., and D. Zacharova. 1966. Potassium contractures in single muscle fibres of the crayfish. J. Physiol. (London). 186: 596. 